\title{
A case of solitary kidney with duplex collecting systems and renal vascular variants in an adult male cadaver
}

\author{
M.S. Salimy, G.A. Luiselli, M. Yuen, R.C. Healy, S.G. Shah, E.L. Giannaris, M. Das, A.E. Wink(1) \\ University of Massachusetts Medical School, Worcester, MA, United States
}

[Received: 28 April 2020; Accepted: 7 July 2020; Early publication date: 29 July 2020]

\begin{abstract}
We describe a unique solitary kidney with duplex collecting system and vascular variation observed in an 86-year-old white male formaldehyde- and phenol-fixed cadaver during routine academic dissection. The left renal fossa was empty with an intact adrenal gland, and the right renal fossa contained a fused renal mass with apparent polarity between the superior and inferior regions and two renal pelves converging into a single ureter. There were three right renal arteries supplying the renal mass; the superior and middle arteries were noted to be postcaval and the inferior artery was precaval. There were also two right renal veins draining into the inferior vena cava and following a regional distribution with the superior vein draining the inferior portion of the renal mass. Despite generally being asymptomatic, the detection of renal anatomical variants is clinically important for appropriate patient management and surgical interventions. (Folia Morphol 2021; 80, 3: 722-725)
\end{abstract}

Key words: renal artery, renal vein, renal pelvis, anatomic variation, multiple renal arteries, multiple renal veins

\section{INTRODUCTION}

The kidneys form between weeks 4 and 12 of development. Congenital solitary kidney (CSK), also known as unilateral renal agenesis, is the failure of one kidney to develop. The estimated prevalence of CSK based on autopsy study is 1 in 1000 cases, occurring slightly more frequently in males and on the left side [20]. CSK is typically asymptomatic, but can be associated with contralateral vesicoureteral reflux, hydronephrosis, hydroureteronephrosis, proteinuria, and genital anomalies $[15,20]$.

Each kidney is typically supplied by a single renal artery arising from the abdominal aorta and one renal vein draining into the inferior vena cava (IVC). Variation in the origin, number, course, division, and penetration of renal arteries is common [15]. The estimated incidence of additional renal arteries arising from the aorta is between $25 \%$ and $30 \%$ [22, 19], and this incidence varies among populations [7]. Variation in the number of renal veins is less common than in the renal arteries [22]; one study of multiple renal veins showed a prevalence of $21.6 \%$ on the right side [4].

We report a unique case of a solitary kidney with two renal pelves draining into a single ureter with accompanying vascular variations.

\section{CASE REPORT}

During academic dissection of a formaldehydeand phenol-fixed 86-year-old white male cadaver, we 


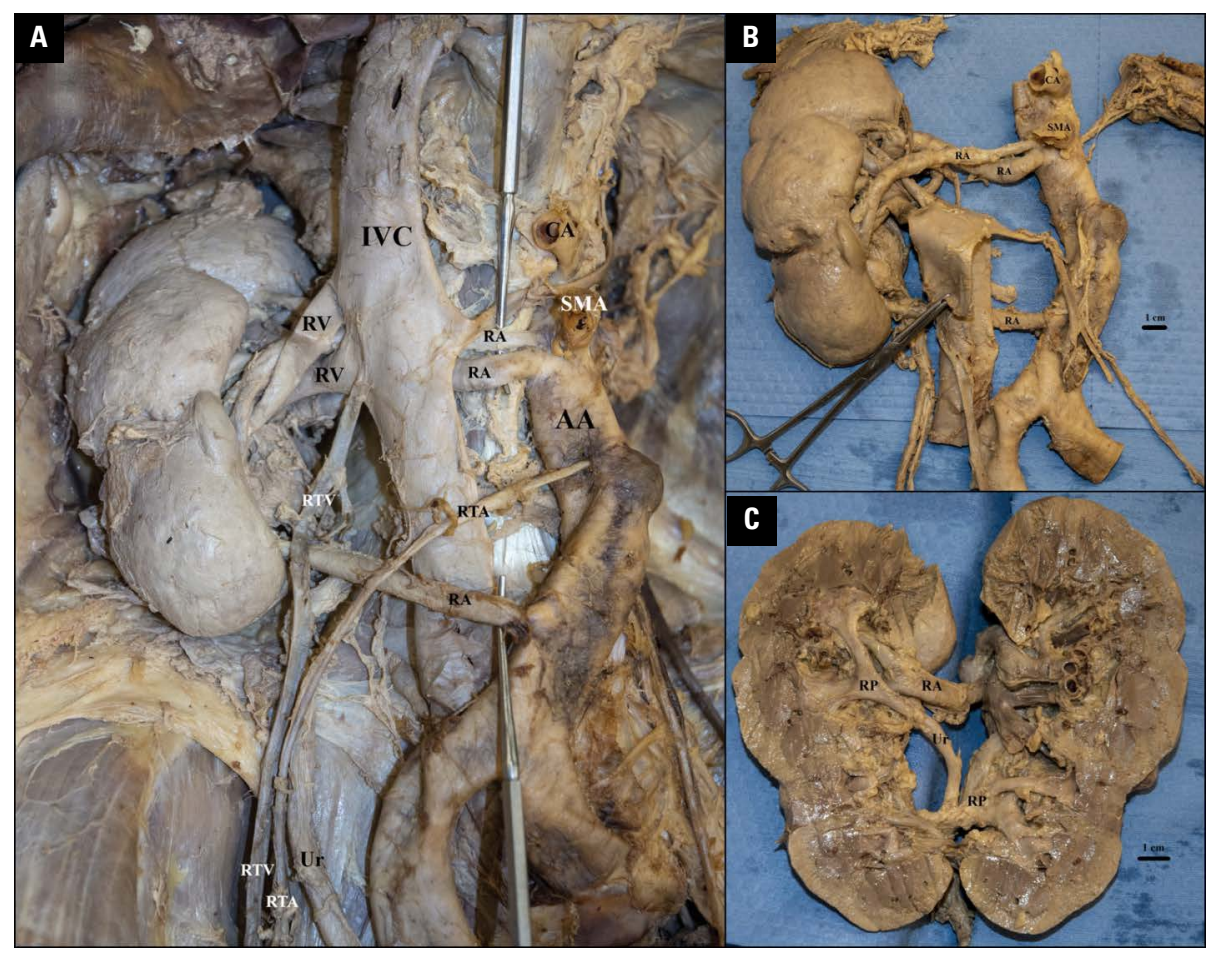

Figure 1. Solitary kidney with dual collecting systems and vascular variation in an adult cadaver. A. In-situ photograph of the renal mass and associated vasculature; B. Ex-situ photograph of the renal mass and associated vasculature; the inferior vena cava (IVC) has been reflected to demonstrate the course of the two cranial renal arteries; C. Sagittal section of the renal mass demonstrating two renal pelves converging into a single ureter; AA — abdominal aorta; CA — coeliac artery; RA — renal artery; RP — renal pelvis; RTA — right testicular artery; RTV — right testicular vein; RV — renal vein; SMA — superior mesenteric artery; $\mathrm{Ur}$ — ureter.

noted an empty left renal fossa. The left adrenal gland was present and its vascular supply arose from the abdominal aorta and drained into the IVC. The left testicular vein was not preserved during dissection and its drainage could not be confirmed. The left testicular artery arose from the abdominal aorta. In the right renal fossa, we exposed an enlarged renal mass (Fig. 1), measuring approximately $16 \mathrm{~cm}$ from the superior to inferior poles and $5.5 \mathrm{~cm}$ from the lateral to medial borders. The renal hilum was below $L 1$, the expected vertebral level. Upon removal of the capsule, we observed a protrusion on the anterior inferior pole of the renal mass.

Further dissection revealed two distinct medially-oriented renal pelves - one draining the upper portion of the kidney and one draining the lower portion of the kidney. Two distinct tributaries converged into a single ureter (Fig. 1C), which descended to the bladder. No additional ureters were encountered in the dissection, and the bladder was not preserved sufficiently to detect the ureteric orifice(s).

Three right renal arteries originated from the abdominal aorta and we refer to them as they arose from the aorta: superior, middle, and inferior. The superior and middle arteries were postcaval and arose from the aorta at approximately the L2 vertebral level, and the inferior artery was precaval and arose from the aorta at approximately the L4 vertebral level. The superior artery crossed anteriorly to the middle artery and bifurcated prior to entering the inferior hilum to supply the more anterior and inferior portion of the kidney. The middle artery crossed posterior to the superior artery and bifurcated into an anterior and posterior branch. The anterior branch gave rise to two polar arteries supplying the superior pole of the kidney before entering the hilum to supply the anterior aspect of the superior (more posterior) portion of the kidney. The posterior branch of the middle artery was distributed to the posterior aspect of the superior portion of the kidney. The inferior artery entered the inferior hilum to supply the anterior inferior portion of the kidney. We identified two right renal veins draining into the IVC. The superior vein crossed anterior to the inferior vein and drained the inferior portion of the kidney, while the inferior vein drained the superior portion. The sagittal dissection of the kidney precluded any attempt to trace the vessels more distally. 


\section{DISCUSSION}

We report a unique case of CSK with duplex collecting system and atypical vasculature (i.e., three right renal arteries and two right renal veins) in an elderly male cadaver. There has been one other reported case of CSK with multiple renal arteries in a female patient with uterine didelphys [15]. This report did not comment on the renal veins, renal pelves, or ureters. The combination of bifid ureters or duplex collecting systems and renal vascular variants (multiple renal arteries and/or renal veins) has been previously reported, but with both kidneys present $[2,10,21]$. The combination of variants described in our report, namely CSK, multiple renal arteries and veins, and two renal pelves converging into a single ureter, has not been reported previously to our knowledge.

An alternative explanation for this morphology is cross-fused renal ectopia (CFRE) with a single ureter. This phenomenon that has been described elsewhere in various contexts $[8,9,12]$; however, these reports exhibited characteristics not seen in our case, namely "pancake morphology", anterior orientation of the renal pelves, and atypical location of the renal mass. The presence of multiple renal vessels is also common in cross-fused renal ectopia [11, 16-18], with many cases reporting atypical origins of the renal arteries, such as the common iliac artery [7]. We report all renal arteries arising from the right side of the abdominal aorta. In CFRE with a single ureter, a scar for the second ureteric orifice is still present on the internal surface of the urinary bladder [8]. The urinary bladder was not preserved sufficiently to detect a second ureteric orifice on the left side; thus, we cannot confirm that our case is one of CFRE. Nonetheless, many of the clinical implications and possible complications of CFRE are similar to those related to general anomalies of upper urinary tract anatomy $[13,18]$.

Ureteral duplication has a reported frequency of $0.6-1.0 \%$ [1]. The ureteric bud, an extension of the mesonephric duct, is the precursor to the ureter. The ureteric bud penetrates the primitive kidney by day 32 of development and elongates and bifurcates to form the ureter, renal pelvis, and major and minor calyces by week 7 . The duplicated pelvis and partially bifid ureter described here could be the result of early bifurcation of the ureteric bud. Bifid ureters are typically asymptomatic, though recurrent urinary infections, flank pain, incontinence, and haematuria may arise as a result of stagnation or reflux of urine from one collecting system into another $[5,21]$.

Clinicians should be aware of these renal anomalies and understand that genitourinary complications may arise from them. There may also be associations between urinary tract anomalies and anomalies of the cardiovascular, central nervous, genital tract, skeletal system, and the gastrointestinal tract. Despite requiring a unique surgical approach, bifid ureters do not affect the complication rate or outcomes of renal transplantation [1].

We also report vascular variation in this donor specimen: two twisting, more cranially-located renal arteries and one additional caudal, precaval renal artery. During development, the kidneys are supplied by transient aortic branches. As the kidneys ascend during weeks six through nine of gestation, these transient branches disintegrate and reform repeatedly to accommodate the ascent. The final pair of arteries to form becomes the renal arteries. If one or more pairs of transient renal arteries fail to regress, they remain as accessory renal arteries. The presence of additional renal arteries can also be explained by genetic background, oxygenation, and haemodynamic changes [3].

Co-occurrence of renal artery and renal vein variations is likely: Cinar and Türkvatan found an association of renal artery and renal vein variation in $15.5 \%$ of 504 patients [4]. The renal veins are formed by the anastomosis of the supracardinal and subcardinal segments of the primitive IVC. Dorsal and ventral renal veins form, but the dorsal typically regresses and the ventral vein persists as the renal vein [14]. Persistence of the dorsal renal vein could account for the two renal veins seen in this case.

Renal vascular variations are typically asymptomatic but may have clinical symptoms such as ischaemia or hypertension [21]. Multiple renal vessels also create surgical challenges during nephrectomy and during aortic reconstruction surgery. Vascular surgeons should be prepared to preserve or revascularise the anomalous renal arteries [6]. In addition, these variants put patients at risk for catastrophic bleeding, so it is crucial for urologists and pelvic surgeons to be vigilant for variant vasculature [9]. Angiography is recommended prior to any surgical procedure involving renal vasculature. 


\section{CONCLUSIONS}

Congenital solitary kidney is a renal anomaly that may be asymptomatic throughout life and remain undetected until incidental finding on autopsy, or it may present with genitourinary symptoms, such as vesicoureteral reflux. Physicians should consider the possibility of CSK in the context of unexplained genitourinary complications and conduct imaging studies to identify such cases and prevent further complications. As seen in our case and another report [15], variations in arterial supply and venous drainage is common in CSK, and variation in ureteric anatomy is also possible. If a patient with CSK undergoes invasive surgery, it is important for the surgeon to plan for potential anatomical and vascular variations.

\section{Acknowledgements}

We would like to thank the anatomical donors for their contribution to our education. We also acknowledge Ms. Amanda Collins, Dr. Yasmin Carter, and Ms. Elizaveta Reznichenko for their assistance with the dissection and preparation of the donor specimen and Ms. Charlene Baron for preparation of the images.

Conflict of interest: None declared

\section{REFERENCES}

1. Alberts VP, Minnee RC, van Donselaar-van der Pant KAMI, et al. Duplicated ureters and renal transplantation: a case-control study and review of the literature. Transplant Proc. 2013; 45(9): 3239-3244, doi: 10.1016/j.transproceed.2013.06.012, indexed in Pubmed: 24182792.

2. Chakravarthi KK, Karuneswari DP, Uma MN. Congenital unilateral double renal pelvis and double ureters associated with triple renal veins and left retro aortic renal vein. Int J Chem Life Sci. 2013; 2(5): 1159-1162.

3. Chawla K, Gupta R, Singh HJ, et al. Bilateral bifid ureter with unilateral renal vasculature variations. Surg Radiol Anat. 2014; 36(4): 393-396, doi: 10.1007/s00276-0131170-9, indexed in Pubmed: 23873247.

4. Çınar C, Türkvatan A. Prevalence of renal vascular variations: Evaluation with MDCT angiography. Diag Interv Imaging. 2016; 97(9): 891-897, doi: 10.1016/j. diii.2016.04.001.

5. Das S, Dhar P, Mehra RD. Unilateral isolated bifid ureter - a case report. J Anat Soc India. 2001; 50(1): 43-44.

6. de Virgilio C, Gloviczki P, Cherry KJ, et al. Renal artery anomalies in patients with horseshoe or ectopic kidneys: the challenge of aortic reconstruction. Cardiovasc Surg. 1995; 3(4): 413-420, doi: 10.1016/0967-2109(95)94161-o, indexed in Pubmed: 7582997.

7. Gulas E, Wysiadecki G, Cecot T, et al. Accessory (multiple) renal arteries - Differences in frequency according to population, visualizing techniques and stage of morphological development. Vascular. 2016; 24(5): 531-537, doi: 10.1177/1708538116631223, indexed in Pubmed: 26945775.

8. Horai K, Naito M, Yakura T, et al. A case of pancake kidney with a single ureter in the retroperitoneal space. Anat Sci Int. 2018; 93(4): 563-565, doi: 10.1007/s12565-018-04421, indexed in Pubmed: 29777511.

9. Kanchan T, Murlimanju BV, Saralaya VV. Pancake kidney with a single ureter: a rare incidental observation at autopsy. Anat Sci Int. 2017; 92(1): 142-146, doi: 10.1007/ s12565-016-0329-y, indexed in Pubmed: 26848753.

10. Kannabathula A, Rai G, Singh KC, et al. Unilateral double pelvis bifid ureter associated with multiple variations of renal vessels. Int J Anat Res. 2016; 4(4.2): 3100-3104, doi: 10.16965/ijar.2016.412.

11. Kaufman MH, Findlater GS. An unusual case of complete renal fusion giving rise to a 'cake' or 'lump' kidney. J Anat. 2001; 198(Pt 4): 501-504, doi: 10.1046/j.14697580.2001.19840501.x, indexed in Pubmed: 11327213.

12. Khanduri S, Tyagi E, Yadav VK, et al. Crossed fused renal ectopia with single ureter and single renal vein: a rare case. Cureus. 2019; 11(1): e3914, doi: 10.7759/cureus.3914, indexed in Pubmed: 30931185.

13. Loganathan AK, Bal HS. Crossed fused renal ectopia in children: a review of clinical profile, surgical challenges, and outcome. J Pediatr Urol. 2019; 15(4): 315-321, doi: 10.1016/j. jpurol.2019.06.019, indexed in Pubmed: 31331806.

14. Mathews R, Smith PA, Fishman EK, et al. Anomalies of the inferior vena cava and renal veins: embryologic and surgical considerations. Urology. 1999; 53(5): 873-880, doi: 10.1016/ s0090-4295(99)00007-2, indexed in Pubmed: 10223477.

15. Matusz P, Miclaus GD, Banciu CD, et al. Congenital solitary kidney with multiple real arteries: case report using MDCT angiography. Rom J Morphol Embryol. 2015; 56(2 Suppl): 823-826.

16. Miclaus GD, Pupca G, Gabriel A, et al. Right lump kidney with varied vasculature and urinary system revealed by multidetector computed tomographic (MDCT) angiography. Surg Radiol Anat. 2015; 37(7): 859-865, doi: 10.1007/ s00276-014-1390-7, indexed in Pubmed: 25380828.

17. Palit S, Datta AK, Tapadar AA. Rare presentation of rudimentary ectopic right kidney fused to the lower pole of the left with multiple aberrant renal vessel - a case report. J Anat Soc India. 2008; 57(2): 146-150.

18. Pupca G, et al. Left crossed fused renal ectopia L-shaped kidney type, with double nutcracker syndrome (anterior and posterior). Rom J Morphol Embryol. 2014; 55: 1237-1241.

19. Satyapal KS, Haffejee AA, Singh B, et al. Additional renal arteries: incidence and morphometry. Surg Radiol Anat. 2001; 23(1): 33-38, doi: 10.1007/s00276-001-0033-y, indexed in Pubmed: 11370140.

20. Shapiro E, Goldfarb DA, Ritchey ML. The congenital and acquired solitary kidney. Rev Urol. 2003; 5(1): 2-8, indexed in Pubmed: 16985610.

21. Stojadinovic D, Zivanovic-Macuzic I, Sazdanovic P, et al. Concomitant multiple anomalies of renal vessels and collecting system. Folia Morphol. 2020; 79(3): 627-633, doi: 10.5603/FM.a2019.0108, indexed in Pubmed: 31617578.

22. Tubbs RS, Shoja MM, Loukas M. Bergman's Comprehensive Encyclopedia of Human Anatomic Variation. John Wiley \& Sons, Hoboken NJ 2016. 